\title{
PERFECT MAPS OF SYMMETRIZABLE SPACES
}

\author{
HAROLD W. MARTIN
}

\begin{abstract}
It is shown that if $f: X \rightarrow Y$ is a perfect map from a symmetrizable space $X$ onto a space $Y$, then $Y$ is metrizable if and only if $f$ is a coherent map. This fact, together with certain known results, yields the following: Let $f: X \rightarrow Y$ be a perfect map from a Hausdorff symmetrizable space $X$ onto a space $Y$; the following are equivalent: (1) $X$ is metrizable; (2) $f$ is a regular map; (3) $f$ is a coherent map; (4) $Y$ is metrizable.
\end{abstract}

A topological space $X$ is said to be symmetrizable if there exists a nonnegative real valued function $d$ on $X \times X$, called a symmetric, which satisfies the following three conditions: (1) $d(a, b)=0$ if and only if $a=b ;(2) d(a, b)=d(b, a) ;(3)$ a subset $A$ of $X$ is closed if and only if whenever $x \in X-A$, then $d(x, A)>0$.

A function $f: X \rightarrow Y$ from a space $X$ onto a space $Y$ is said to be coherent if the space $X$ is symmetrizable via a symmetric $d$ such that whenever $\left\{a_{n}\right\}$ and $\left\{b_{n}\right\}$ are sequences in $X$ with $d\left(a_{n}, b_{n}\right) \rightarrow 0$ and $f\left(a_{n}\right) \rightarrow y$ in $Y$, then $f\left(b_{n}\right) \rightarrow y$. Coherent maps are closely related to the regular maps of $\mathrm{A}$. Arhangel'skir [1, p. 133]. Every regular map is a coherent map. The extent of coherent maps may be seen in the following, which is not difficult to prove: Let $f: X \rightarrow Y$ be a function from a symmetrizable space $X$ onto a metrizable space $Y$; then, $f$ is continuous if and only if $f$ is a coherent map.

A map is perfect if it is closed, continuous and point inverses are compact, i.e., bicompact, sets.

THEOREM 1. Let $f: X \rightarrow Y$ be a perfect map from a symmetrizable space $X$ onto a space $Y$. Then, $Y$ is metrizable if and only if $f$ is a coherent map.

Proof. Assume that $Y$ is metrizable. Let $\rho$ be a symmetric for $X$ and $d$ be a metric for $Y$. For points $a$ and $b$ in $X$, let $\sigma(a, b)=\rho(a, b)+$ $d(f(a), f(b)) . \sigma$ is a symmetric compatible with the topology for $X$. It is easy to verify that $f$ is a coherent map by virtue of the symmetric $\sigma$.

Presented to the Society, January 28, 1972 under the title Perfect coherent maps of symmetrizable spaces; received by the editors April 10, 1972 and, in revised form, July 31, 1972.

AMS (MOS) subject classifications (1970). Primary 54C10, 54E35; Secondary 54E25.

Key words and phrases. Metrizable space, symmetrizable space, symmetric, $G_{\delta^{-}}$ diagonal, perfect map, coherent map, regular map.

(C) American Mathematical Society 1973 
To prove the converse, assume that $f$ is a coherent map by virtue of a symmetric $\rho$ for the space $X$. For $a, b \in Y$, define $d(a, b)=\rho\left(f^{-1}(a), f^{-1}(b)\right)$. We shall show that the space $Y$ is symmetrizable via $d$. Clearly, $d(a, b)=$ $d(b, a)$ for all $a, b \in Y$. That $d(a, b)=0$ if and only if $a=b$ follows easily from the fact that $f$ is a coherent map and $Y$ is a $T_{1}$ space. Let $A$ be a closed subset of $Y$ and $y \in Y-A$. If $d(y, A)=0$, then $\rho\left(f^{-1}(y), f^{-1}[A]\right)=0$ and there would exist sequences $\left\{b_{n}\right\}$ in $f^{-1}(y)$ and $\left\{a_{n}\right\}$ in $f^{-1}[A]$ such that $\rho\left(a_{n}, b_{n}\right) \rightarrow 0$; since $f\left(b_{n}\right) \rightarrow y$, we would have $f\left(a_{n}\right) \rightarrow y$, contradicting the closedness of $A$. Consequently, $d(y, A)>0$. Finally, assume that $B$ is not a closed subset of $Y$. Since $f$ is a quotient map, $f^{-1}[B]$ is not a closed subset of $X$. Therefore, there exists $x \in X-f^{-1}[B]$ such that $\rho\left(x, f^{-1}[B]\right)=$ 0 . Then $d(f(x), B)=0$, and we have completed the proof that the space $Y$ is symmetrizable via $d$.

Let $A$ be a compact subset of $Y$ and $B$ be a closed subset of $Y$ such that $d(A, B)=0$. Then $\rho\left(f^{-1}[A], f^{-1}[B]\right)=0$ and since $f^{-1}[A]$ is compact, there exists $x \in f^{-1}[A]$ and sequences $\left\{a_{n}\right\}$ in $f^{-1}[A]$ and $\left\{b_{n}\right\}$ in $f^{-1}[B]$ such that $a_{n} \rightarrow x$ and $\rho\left(a_{n}, b_{n}\right) \rightarrow 0$. Since $f$ is a coherent map, we have $f\left(b_{n}\right) \rightarrow f(x)$. Since $B$ is closed, it follows that $f(x) \in B$ so that $A$ and $B$ are not disjoint. Hence, if $A$ and $B$ are disjoint subsets of $Y$ with $A$ compact and $B$ closed, then $d(A, B)>0$. By Theorem 2 of [3], $d$ is a coherent distance function. The metrizability of $Y$ now follows by a theorem of Niemytzki and Wilson [5], [8], completing the proof.

If a Hausdorff space $X$ maps perfectly onto a metrizable space, then $X$ is metrizable if and only if $X$ has a $G_{\delta}$-diagonal [2], [6]. Recall also that the perfect image of a metrizable space is metrizable [4], [7]. These facts, together with Theorem 1, yield the following:

THEOREM 2. Let $f: X \rightarrow Y$ be a perfect map from a symmetrizable Hausdorff space $X$ onto a space $Y$. The following are equivalent:

(1) $X$ is metrizable.

(2) $f$ is a regular map.

(3) $f$ is a coherent map.

(4) $Y$ is metrizable.

Proof. Assume that $X$ is metrizable. Then $Y$ is metrizable. It follows that $f$ is regular [1, p. 134], so that (1) implies (2). As seen in [3], every regular map is coherent, so that (2) implies (3). (3) implies (4) by Theorem 1. Finally, assume that $Y$ is metrizable. Then $X$ is regular; any regular space which maps onto a first countable space by a closed map with first countable point inverses is itself first countable, i.e., $X$ is a first countable space. This completes the proof since any first countable symmetrizable Hausdorff space has a $G_{\delta}$-diagonal. 
The Hausdorff condition on $X$ in Theorem 2 cannot be completely removed since there exist non-Hausdorff compact symmetrizable spaces.

\section{REFERENCES}

1. A. V. Arhangel'skiĭ, Mappings and spaces, Uspehi Mat. Nauk 21 (1966), no. 4 (130), 133-184=Russian Math. Surveys 21 (1966), no. 4, 115-162. MR 37 \#3534.

2. C. Borges, On stratifiable spaces, Pacific J. Math. 17 (1966), 1-16. MR 32 \#6409.

3. $\mathrm{H}$. Martin, Metrization of symmetric spaces and regular maps, Proc. Amer. Math. Soc. 35 (1972), 269-274.

4. K. Morita and S. Hanai, Closed mappings and metric spaces, Proc. Japan Acad. 32 (1956), 10-14. MR 19, 299.

5. V. Niemytzki, On the third axiom of metric spaces, Trans. Amer. Math. Soc. 29 (1927), 507-513.

6. A. Okuyama, On metrizability of M-spaces, Proc. Japan Acad. 40 (1964), 176-179. MR 29 \#5222.

7. A. H. Stone, Metrizability of decomposition spaces, Proc. Amer. Math. Soc. 7 (1956), 690-700. MR 19, 299.

8. W. A. Wilson, On semi-metric spaces, Amer. J. Math. 53 (1931), 361-373.

Department of Mathematics, University of Pittsburgh, Pittsburgh, PennSYLVANIA 15213 